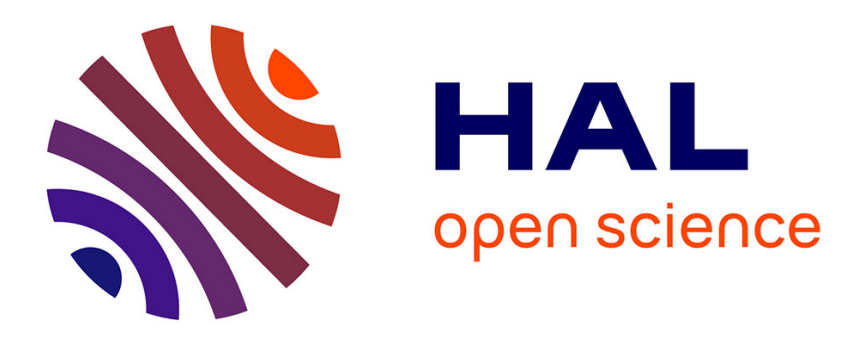

\title{
Probing interfacial dynamics of water in confined nanoporous systems by NMRD \\ Pierre Levitz
}

\section{To cite this version:}

Pierre Levitz. Probing interfacial dynamics of water in confined nanoporous systems by NMRD. Molecular Physics, 2018, 117 (7-8), pp.952-959. 10.1080/00268976.2018.1527960 . hal-02374464

\section{HAL Id: hal-02374464 https://hal.sorbonne-universite.fr/hal-02374464}

Submitted on 21 Nov 2019

HAL is a multi-disciplinary open access archive for the deposit and dissemination of scientific research documents, whether they are published or not. The documents may come from teaching and research institutions in France or abroad, or from public or private research centers.
L'archive ouverte pluridisciplinaire HAL, est destinée au dépôt et à la diffusion de documents scientifiques de niveau recherche, publiés ou non, émanant des établissements d'enseignement et de recherche français ou étrangers, des laboratoires publics ou privés. 
Probing interfacial dynamics of water in confined nanoporous systems by NMRD

Pierre Levitz

PHENIX Laboratory, Sorbonne Université and CNRS. Paris, France

Corresponding contributor: e-mail : pierre.levitz@sorbonne-universite.fr

MOLECULAR PHYSICS

Volume: 117

Issue: $7-8$

Pages: $952-959$

Special Issue: SI

DOI: $10.1080 / 00268976.2018 .1527960$

Published: APR 182019 


\begin{abstract}
The confined dynamics of water molecules inside a pore involves an intermittence between adsorption steps near the interface and surface diffusion and excursions in the pore network. Depending of the strength of the interaction in the layer(s) close to the surface and the dynamical confinement of the distal bulk liquid, exchange dynamics can vary significantly. The average time spent in the surface proximal region (also called the adsorption layer) between a first entry and a consecutive exit allows estimating the level of "nanowettablity" of water. As shown in several seminal works, NMRD is an efficient experimental method to follow such intermittent dynamics close to an interface.

In this paper, the intermittent dynamics of a confined fluid inside nanoporous materials is discussed. Special attention is devoted to the interplay between bulk diffusion, adsorption and surface diffusion on curved pore interfaces. Considering the nano or meso length scale confinement of the pore network, an analytical model for calculating the intradipolar spin-lattice relaxation dispersion curves is proposed. In the low frequency regime $(50 \mathrm{KHz}-100 \mathrm{MHz})$, this model is successfully compared with numerical simulations performed using a 3D-off lattice reconstruction of Vycor glass. Comparison with experimental data available in the literature is finally discussed.
\end{abstract}

\title{
Keywords:
}

NMR relaxometry, porous media, adsorption, intermittent dynamics, confinement; water dynamics, Vycor porous glass.

\section{Introduction}

Water confined in the vicinity of mineral surfaces is ubiquitously encountered in numerous industrial nanoporous materials as well as in geological materials and biological tissues. The level of saturation and the specific properties of confined water strongly influence fluid transport and the rheological and poromechanical characteristics of these materials [1]. Various implications in catalysis, nanofiltration, life cycle and durability of various systems have to be considered.

Water confinement in restricted geometry involves a proximal (or adsorption) layer and a distal region characterized by a bulky confinement in the pore network. As shown in Fig 1 , the molecular trajectory can be described as an alternate succession of surface adsorption steps followed by excursions in the confined bulk, generating a new relocation on the surface. As a result, the transport process appears as an intermittent dynamics sensitive to the interaction of the vicinal fluid with the interface and to the nature of bulk confinement [2-5]. 
Neutron spin echo or quasi-elastic scattering [6] can be used to follow local molecular dynamics inside the pore network or nearby the interfacial region. At very long time and large distance (above $1 \mathrm{~ms}$ and $1 \mu \mathrm{m}$ ), macroscopic experiments or NMR pulsed field gradient spin echo experiments could be used. At the mesoscopic scale, mainly, for a time window ranging from few ns to some $\mu$ s, a promising way to follow the slow dynamics of an embedded fluid is to analyze the dispersion of the nuclear spin-lattice relaxation rate of the fluid using field cycling nuclear magnetic relaxation dispersion technique (NMRD) [7,8].

In this paper, we analyse the intermittent dynamics of a confined fluid inside nanoporous materials. These nanoporous networks are in general multiconnected systems. We focus on the interplay between bulk diffusion, adsorption and surface diffusion on curved pore interfaces. In section 2, we present some general properties of molecular intermittent dynamics. In section 3, we analyse how NMRD allows probing such dynamics with special emphasis on the role of pore surface curvature. In section 4, we compare our analytical model with numerical simulations performed on a toy $3 \mathrm{D}$ pore network mimicking Vycor glass $[9,10]$. Finally, in section 5, we provide a comparison with experimental NMRD data performed on Vycor glass [8,11].

\section{Intermittent dynamics of water in nanoporous systems}

As shown in Fig 2, the time dependence of the intermittent dynamics of a confined fluid near a pore interface can be analysed using two density probability distribution (p.d.f.). The first p.d.f [4], $\Psi_{\mathrm{A}}(\mathrm{t})$ characterizes the way according to which an adsorbed molecule is released in the bulk. $\Psi_{\mathrm{A}}(\mathrm{t})$, is the distribution of adsorption time separating an entrance in the proximal zone and its first desorption to the distal region. The first moment of $\Psi_{\mathrm{A}}(\mathrm{t}), \tau_{\mathrm{A}}$, is the average time spent in the adsorption region between a first entry and the consecutive first exit. The second important p.d.f, referred to as $\Psi_{\mathrm{B}}(\mathrm{t})$, is the bridge statistics that provides the time distribution between a desorption event and the next first possible reencounter within the proximal zone. The first moment of $\Psi_{\mathrm{B}}(\mathrm{t})$ is noted $\tau_{\mathrm{B}}$. Various analytical expressions of $\Psi_{\mathrm{B}}(\mathrm{t})$ were discussed in the literature especially for what we call a "open surface" such as flat [3,12], rough [13] or external cylindrical interfaces [14]. In such cases, bridge statistics of a Brownian motion exhibit an algebraic tail at long time, evolving as $\mathrm{c} / \mathrm{t}^{1+\mu}$ with $0<\mu<1$. On the contrary for nanoporous 
networks, one generally observes an exponential cut-off, suppressing a large part of the algebraic tail of $\Psi_{\mathrm{B}}(\mathrm{t})$, at long time. This point was checked recently by extended molecular dynamics simulation of confined liquid water filling either a hydrophilic $\mathrm{SiO}_{2}$ or a hydrophobic carbon nanopore [5].

When there is an exchange between a proximal (or adsorption) layer and a distal region characterized by a bulky confinement in the pore network, special attention must be devoted to detailed balance conditions that also define the level of adsorption on the interface. In fact, when a molecule hits the pore wall, its probability to be adsorbed is not unity but a probability $\mathrm{p} \leq 1$. The p.d.f., $\Psi_{\mathrm{R}}(\mathrm{t})$, associated to the relocation statistics is related to $\Psi_{\mathrm{B}}(\mathrm{t})$ according to:

$$
\widetilde{\Psi}_{R}(\omega)=\frac{p \widetilde{\Psi}_{B}(\omega)}{1-(1-p) \widetilde{\Psi}_{B}(\omega)}
$$

where $\widetilde{\Psi}_{R}(\omega)$ and $\widetilde{\Psi}_{B}(\omega)$ are the time Fourier transforms of $\Psi_{R}(t)$ and $\Psi_{B}(t)$, respectively. It is straightforward, using Eq 1, to show that a relocation in the pore space from a first desorption to a first readsorption takes an average time $\tau_{R}=\tau_{B} / p$. p and $\tau_{A}$, then define the level of adsorption. Using ergodicity hypothesis, the detailed balance condition is written as:

$$
\tau_{A}=\frac{\tau_{B}}{p}\left[\frac{f_{a d s}}{1-f_{a d s}}\right]
$$

$f_{a d s}$ is the fraction of molecules located inside the adsorption layer and can be written as:

$$
f_{a d s}=S_{v} \delta \Gamma_{s}
$$

where $S_{v}$ is the surface area normalized to the pore volume, $\delta$ the thickness of the adsorption layer and $\Gamma_{\mathrm{s}}$, its molecular compacity compared to the bulk confined fluid.

The time evolution can be described using an indicator function $I(t)$ equals to one in the adsorption state and zero in the confined bulk. This random signal is associated to probability distribution functions $\Psi_{\mathrm{A}}(\mathrm{t})$ and $\Psi_{\mathrm{R}}(\mathrm{t})$. A way to characterize the statistical nature of this intermittent dynamics is to compute the average time auto-correlation function of the random signal I(t) [4]. 


$$
C(\tau)=\overline{\langle I(\tau) \cdot I(0)\rangle} / \eta_{a}
$$

With $\eta_{a}=\tau_{A} /\left(\tau_{A}+\tau_{R}\right)$. The bar is associated to the average over all initial times " 0 " along a trajectory. The two brackets stand for the ensemble average over all possible trajectories. It is straightforward to show that $\mathrm{C}(0)=1$ and. $C(+\infty)=\eta_{a}$. As discussed elsewhere $[4,5]$, it is convenient to compute the spectral density $\tilde{J}(\omega)$ of the noise $I(t)$. $\tilde{J}(\omega)$ is the time Fourier Transform (FT) of C( $\tau)$. Such a computation can be done easily if a statistical independence between successive adsorption steps and relocation periods is assumed. Under this hypothesis and following [4], we get:

$$
\tilde{J}(\omega)=\frac{2}{\tau_{A} \omega^{2}} \operatorname{Re} a l\left[\frac{\left(1-\tilde{\Psi}_{A}(\omega)\right)\left(\left(1-\tilde{\Psi}_{R}(\omega)\right)\right.}{1-\tilde{\Psi}_{A}(\omega) \cdot \tilde{\Psi}_{R}(\omega)}\right]
$$

It is worth noting that the integral of this spectral density is normalized to unity.

\section{Intermittence in nanoporous systems and NMRD}

As first shown by Kimmich et al [7,8], it is possible to follow by NMRD the dynamics of a molecule near a solid interface through the evolution of the fluctuating intra-dipolar magnetic interaction $\mathrm{H}(\mathrm{t})$. This interaction at low magnetic field is essentially related to the rotational dynamics of water molecules. It was shown that $\mathrm{H}(\mathrm{t})$ is mainly sensitive (in the slow dynamics limit) to the time evolution of the surface director probed by the molecule during its self-diffusion [15]. Such type of magnetic fluctuation was first discussed by Kimmich et al [2] by introducing the concept of reorientation mediated by translation displacement (RMTD). Looking at low frequency domain of fluctuation (coarse grain picture), $\mathrm{H}(\mathrm{t})$ takes a series of values during each adsorption step directly related to the local surface orientation and another value in the confined bulk phase. Curvature, persistence length and roughness of a surface can then be probed by following the intermittent dynamics close to it. A schematic evolution of $\mathrm{H}(\mathrm{t})$ is shown in Fig. 3. In general, and compared to Fig. 2, H(t) can continuously evolve during an adsorption step and get different values from one adsorption event to another one. This evolution is mainly driven by the curvature field of the poral interface coupled to molecular surface diffusion.

As for $\mathrm{I}(\mathrm{t})$, a sensitive way to probe temporal fluctuations of $\mathrm{H}(\mathrm{t})$ is to look at the spectral

density of this magnetic noise, noted $\tilde{J}_{H}(\omega)$. This noise induces a nuclear magnetic 
relaxation process at the Larmor frequency $\omega$ or $\mathrm{f}=\omega / 2 \pi$. Using field cycling NMR spectroscopy, the related spin-lattice relaxation rate $\mathrm{R}_{1}(\omega)$ can be measured over a large range of frequencies, mainly from few $\mathrm{kHz}$ to several tens of $\mathrm{MHz}$. This frequency range allows probing correlation times ranging from $1 \mathrm{~ns}$ to tenths of $\mu \mathrm{s}$. In the following, we will use a normalization of the spectral density such as:

$$
\int_{\Omega(\omega)} \tilde{J}_{H}(\omega) d \omega=1
$$

Without considering the contribution of the confined fluid in the bulk (which is essentially constant at low magnetic field) but considering the intermittence process only, the spin-lattice relaxation rate is written after the former normalization as [16]:

$$
\mathrm{R}_{1}^{N}(\omega)=\tilde{J}_{H}(\omega)+4 \tilde{J}_{H}(2 \omega)
$$

\section{Interplay between surface curvature and molecular intermittence.}

Looking at Fig. 3, H(t) can be written as $\mathrm{H}(\mathrm{t})=(\mathrm{O}(\mathrm{t}) \cdot \mathrm{I}(\mathrm{t}))$. $\mathrm{O}(\mathrm{t})$ is the contribution of the surface curvature of the poral interface coupled with molecular surface diffusion. Following former analysis [4], the autocorrelation of $\mathrm{H}(\mathrm{t})$ can be approximated as

$$
\overline{\langle H(\tau) \cdot H(0)\rangle}=\overline{\langle(O(0) . I(0)) .(O(\tau) \cdot I(\tau))\rangle} \approx \overline{\langle O(0) . O(\tau)\rangle} \overline{\langle I(0) . I(\tau)\rangle}
$$

Then the spectral density of $\mathrm{H}(\mathrm{t})$ reads

$$
\tilde{J}_{H}(\omega) \approx \tilde{C}_{O}(\omega) * \tilde{J}(\omega)
$$

$\tilde{C}_{O}(\omega)$ is the Fourier transform of $C_{O}(\tau)=\overline{\langle O(0) . O(\tau)\rangle}$. * stands for the convolution operator. The constant of proportionality in Eq. 9 is defined according to eq. (6). $C_{O}(\tau)$ takes into account the fluctuation of the surface director and its time correlation during a surface diffusion without desorption. In the laboratory framework and following various works of Halle [17], we propose to write

$$
C_{O}(\tau)=\overline{\left\langle Y_{2}^{m}\left(\Omega_{s}(0)\right) \cdot Y_{2}^{m^{*}}\left(\Omega_{s}(\tau)\right)\right\rangle}
$$

with $\mathrm{m}=1 . \Omega_{s}(\tau)$ are the Euler angles between the constant magnetic field axis and the surface director where the molecule is located at time $\tau$. If the interfacial medium is statistically isotropic, the evolution of $C_{O}(\tau)$ is similar for $\mathrm{m}=0,1,2$. For a set of randomly oriented flat or cylindrical surfaces, $C_{O}(\tau)$ is a constant in time and $\tilde{C}_{O}(\omega)=\delta(\omega)$. Then, 


$$
\tilde{J}_{H}(\omega) \approx \tilde{J}(\omega)
$$

Reciprocally for a diffusion inside the adsorption layer without desorption, $I(\tau)$ is a constant and $\tilde{J}(\omega)=\delta(\omega)$. Then $\tilde{J}_{H}(\omega) \approx \tilde{C}_{0}(\omega)$.

\section{Numerical simulations versus analytical model}

In order to check the level of accuracy of our analytical analysis, we have performed a series of numerical simulations of the molecular intermittent dynamics inside a toy model mimicking a Vycor glass. This material was previously investigated using transmission electron microscopy (TEM), small angle $\mathrm{X}$ ray and neutron scattering (SAXS) and was free of paramagnetic impurity as shown by EPR at 4K [9]. As described elsewhere [10], a 3D off-lattice reconstruction of this pore network, defined by a correlated Gaussian field, allows obtaining a numerical representation that concurs with SAXS data, pore and solid chord length distributions, porosity (0.3), surface area normalized to the full volume (100 $\mathrm{m}^{2} / \mathrm{cm}^{3}$ ) and pore size distribution (average pore radius of $3 \mathrm{~nm}$ ) of the chosen Vycor porous glass. A configuration is shown in Fig. 4. The pore network is in white. The edge of the cube is $300 \mathrm{~nm}$. The pore network was divided in two regions: the adsorption layer with an average thickness $\delta=0.3 \mathrm{~nm}$ and the complementary space which is the distal region characterizing bulky confinement. $\Gamma$ s, the surface molecular compacity compared to the bulk confined fluid is set to one. We have simulated the self-diffusion of a fluid molecule inside this pore network. Brownian dynamics, with a time step of $210^{-12} \mathrm{~s}$, was performed. The self-diffusion coefficient of the fluid in distal region, Dbulk, is set according to the Renkin correction [18] taking into account a molecular radius of $0.15 \mathrm{~nm}$ and an average accessible pore radius of $2.7 \mathrm{~nm}$. The chosen value is Dbulk $=1.7410^{-9}$ $\mathrm{m}^{2} / \mathrm{s}$. Each time a molecule hits the interface, it gets adsorbed for a time $\mathrm{t}$ according to the $\operatorname{pdf} \Psi_{\mathrm{A}}(\mathrm{t})$, with the possibility to diffuse inside the adsorption layer. Following ref [19], the self-diffusion coefficient $\mathrm{D}_{\text {surf }}$ is set to $\mathrm{D}_{\text {bulk }} / 3$. We chose an exponential evolution for $\Psi_{\mathrm{A}}(\mathrm{t})$ that is characterized by the average adsorption time $\tau_{\mathrm{A}}$. The $\mathrm{p}$ value is computed according to Eq.2.

In Fig. 5, we show the evolution of the relocation p.d.f. $\Psi_{R}(t)$ with the average adsorption time $\tau_{\mathrm{A}}$. For very short $\tau_{\mathrm{A}}$, its evolution is very close to the one observed for $\Psi_{\mathrm{B}}(\mathrm{t}) . \Psi_{\mathrm{R}}(\mathrm{t})$ evolves first as $\mathrm{t}^{-3 / 2}$ as found for a flat surface [12]. At longer time, an exponential cutoff is observed. These two results are also observed in molecular dynamics simulations 
performed inside a $2 \mathrm{~nm}$ thick $\mathrm{SiO}_{2}$ slit pore [5]. As $\tau_{\mathrm{A}}$ increases, we observe a shift of the curves to higher time and $\Psi_{\mathrm{R}}(\mathrm{t})$ is essentially dominated by an exponential tail, as shown in Fig. 6. Then, the first moment of $\Psi_{R}(t), \tau_{R}$, has the same value as the characteristic time of the decreasing exponential tail. Interestingly enough, we see in Fig.6 that the numerical simulation and the analytical prediction of $\Psi_{\mathrm{R}}(\mathrm{t})$ (using Eqs 1-3 and the reverse Fourier transform of Eq.1) are in very good agreement. As $\tau_{\mathrm{A}}$ increases, $\tau_{R}$ diverges to larger and larger values. Typically, for $\tau_{A}=0.2 n s, \tau_{R}=1.2 \mathrm{~ns}$, and for $\tau_{\mathrm{A}}=100 \mathrm{~ns}, \tau_{\mathrm{R}}=590 \mathrm{~ns}$.

We have computed the spin lattice relaxation rate assuming that the mean relaxation process is mainly due to intramolecular dipolar interactions. In the low frequency domain, we estimate the normalized spin lattice relaxation rate as:

$$
\mathrm{R}_{1}^{c}(\omega)=\tilde{J}_{c}(\omega)+4 \tilde{J}_{c}(2 \omega)
$$

where

$$
\tilde{J}_{c}(\omega) \propto F T\left[\overline{\left\langle\left(I(0) \cdot Y_{2}^{1}\left(\Omega_{s}(0)\right)\right) .\left(Y_{2}^{1^{*}}\left(\Omega_{s}(\tau)\right) \cdot I(\tau)\right)\right\rangle}\right]
$$

with the normalisation

$$
\int_{\Omega(\omega)} \tilde{J}_{c}(\omega) d \omega=1
$$

The term $\overline{\left\langle\left(I(0) \cdot Y_{2}^{1}\left(\Omega_{s}(0)\right)\right) .\left(Y_{2}^{1^{*}}\left(\Omega_{s}(\tau)\right) \cdot I(\tau)\right)\right\rangle}$ is obtained after averaging on a large number of trajectories

In Fig 7, we compare our numerical simulation with two possible models, using either Eqs. 5,6,7,11 where only intermittence is considered (model IT), or Eqs. 5,6,7,9 where intermittence and surface curvature are coupled (model IT-SC). First of all, we observe a good agreement between numerical simulations and model IT-SC whatever the average adsorption time. For short and very short $\tau_{\mathrm{A}}$. (see Fig 7A), IT-SC and IT models evolve in a similar way and close to numerical simulations. After a plateau, the dispersion curve starts decreasing above a frequency on the order of $\left(\tau_{R}+\tau_{A}\right) /\left(2 \pi \tau_{R} \cdot \tau_{A}\right)$. For these small adsorption times, the curvature of the interface does not play an important role in the dispersion curve. Surface diffusion events are too short to build an efficient time surface correlation. As $\tau_{\mathrm{A}}$ increases, we observe a significant discrepancy between the IT model and numerical simulations. For long surface diffusion periods, it is then essential to take into account both intermittence and surface curvature. In parallel we observe a shift of the 
dispersions toward low frequency as $\tau_{\mathrm{A}}$ increases. Again, a plateau emerges for frequencies lower than about $\left(\tau_{R+} \tau_{A}\right) /\left(2 \pi \tau_{R} \cdot \tau_{A}\right)$. Finally, for very long adsorption times (Fig 7D), the two dispersion curves associated to the numerical simulation and the IT-SC model both exhibit a $1 / \mathrm{f}^{1 / 2}$ evolution. Moreover, these two curves are very close to $\tilde{C}_{O}(\omega)$ . As expected for this adsorption regime, we are essentially probing the effect of surface diffusion on the spin-lattice relaxation rate.

It is interesting to look at the evolution of the IT model with increasing average adsorption time. As shown in Fig. 7, the associated dispersion curve rapidly evolves toward a Lorentzian shape. In parallel, as shown in Fig. 6, $\Psi_{\mathrm{R}}(\mathrm{t})$ is dominated by an exponential tail with a characteristic time $\tau_{\mathrm{R}}$. Considering that $\Psi_{\mathrm{A}}(\mathrm{t})$ is also an exponential with an average adsorption time $\tau_{\mathrm{A}}$, it is straightforward using Eq. 5, to show that $\tilde{J}(\omega)$ and $\tilde{J}_{H}(\omega)$ are Lorentzian functions with a characteristic time equal to $\left(\tau_{\mathrm{R} .} \tau_{\mathrm{A}}\right) /\left(\tau_{\mathrm{R}}+\tau_{\mathrm{A}}\right)$.

\section{Comparison with experimental RMND data}

Several experimental NMRD works $[7,8,11]$ were performed to elucidate the confined dynamics of liquid water inside Vycor porous glass. At room temperature, it was shown that the frequency dependences of ${ }^{1} \mathrm{H}$ spin-lattice relaxation rate of water inside this porous material were mainly evolving as $1 / \mathrm{f}^{\alpha}$ with $\alpha$ close to $1 / 2$. Moreover, a very striking property was observed [8]. The exponent of the dispersion curve does not change when the temperature is decreased below the freezing point of the confined liquid. More recently [11], we performed NMRD of both normal and deuterated water saturating Vycor. The sample was previously investigated using transmission electron microscopy (TEM), small angle scattering and neutron scattering [9]. The sample was free of paramagnetic impurities as shown by electron spin resonance spectroscopy. We have obtained similar data as those of the Kimmich 's group [8], as shown in Fig 8. The ${ }^{2} \mathrm{H}$ NMRD of $\mathrm{D}_{2} \mathrm{O}$ saturating the pore network evolves in a similar way as the ${ }^{1} \mathrm{H}$ NMRD. This confirms that the spin-lattice relaxation rate of the proton is essentially dominated by intra-dipolar interactions. Moreover, and as proposed earlier [8], the invariance of the exponent of algebraic dispersion curves with temperature clearly indicates that NMRD at low frequencies is essentially due to a population of molecules diffusing during a long or 
very long time close to the pore surface, in agreement with the numerical and theoretical analysis discussed in the present paper. In this specific regime, relocation dynamics play a minor role. What is probed is related to the correlation of surface curvature inside this multiconnected system during surface diffusion. A different situation is encountered in dilute colloidal systems or for a flat open surface $[4,14]$ where intermittence takes place inside an unconfined volume. In these cases, the existence of algebraic tails at long time, for $\Psi_{\mathrm{B}}(\mathrm{t})$ and $\Psi_{\mathrm{R}}(\mathrm{t})$, is determinant for the analysis of bulk mediated surface diffusion proposed in the seminal work of Bychuk and O'Shaughnessy [3] and in NMRD experiments. Algebraic evolution of the dispersion curves can be observed, even for short value of $\tau_{\mathrm{A}}[4,14]$.

To end this section, we want to provide some additional remarks on the case of confining nanopores where $C_{O}(\tau)$ is a constant in time and $\tilde{C}_{O}(\omega)=\delta(\omega)$. A typical example of such a situation can be provided by a set of randomly oriented cylindrical mesopores. This can be encountered in the case of a powder of mesoporous materials possessing a two-dimensional structure of aligned cylindrical pore channels. In some specific systems of this type, it was experimentally shown that intra-dipolar dispersion curve in the low frequency regime evolves as a Lorentzian [20]. Looking at the result shown in Fig 7D for the IT model, it is then tempting to assign such an evolution to a population of water molecules staying for very long time close to the cylindrical surface. At this level, this is only a guess that requires further extended analysis

\section{Conclusion}

In the first part of this work, we have analyzed some properties of intermittent dynamics of a confined fluid inside a nanoporous porous media. In contast with the case of large macroporous systems or dilute colloidal interfaces, the excursion of molecules in the confined bulk between two adsorption events is limited in length with a specific cut-off linked to the finite size of the pore.

In the second part of this paper, we have discussed how NMRD allows probing the dynamics of confined fluids with a specific emphasis on intra or quadripolar magnetic interactions. The role of pore surface curvature fluctuations is analyzed in relation with the level of molecular interaction with the pore surface. An analytical model of the spinlattice relaxation dispersion curves is proposed and successfully compared with 
numerical simulations performed using a 3D-off lattice reconstruction of Vycor glass. In the third part, a comparison with experimental measurements, available in the literature, was finally performed. It confirms that the low frequency NMRD of water protons inside Vycor is essentially due to a population of molecules staying and diffusing in the adsorption layer (the proximal zone near the pore wall) for a very long time (some $\mu$ s or more). This conclusion is in good agreement with former analysis performed by Kimmich's group [8]. Moreover, our results are in phase with the hypothesis of strongly bounded water molecules at the solid interface. Such a hypothesis was recently indirectly inferred in spontaneous and forced imbibition experiments on water in Vycor [21,22]. The proposed model, involving interplay between molecular intermittence and surface curvature can be used to other porous geometries. It is the subject of ongoing work that will be published later.

\section{Acknowlegments}

It is a pleasure to thank my former colleagues of the NMR group of Ecole Polytechnique, Jean-Pierre Korb and Dominique Petit. I also thank for their dynamism, the new Low Field NMR group of my present laboratory: PHENIX (Sorbonne University, Paris): Anne-Laure Rollet and Guillaume Meriguet.

The authors acknowledge COST Action CA15209 EURELAX "European Network on NMR Relaxometry”, supported by COST (European Cooperation in Science and Technology).... 


\section{References}

[1] C. Alba-Simionesco, B. Coasne, G. Dosseh, G. Dudziak,SM. liwinska-Bartkowiak, J.Phys. Condens. Matter 18, R15 (2006)

[2] R. Kimmich and H.B. Weber, Phys. Rev. B, 47, 788 (1993)

[3] O. V. Bychuk. and B. O. Shaughnessy, J. Phys. II (France), 4, 1135 (1994)

[4] P. Levitz, J. Phys.: Condens. Matter, 17, S4059.13. (2005)

[5] P. Levitz P., P.A., Bonnaud P-A Cazade, R J-M Pellenq and B Coasne, Soft Matter, 9, 8654 (2013)

[6] J. C. Perrin, S. Lyonnard, and F. Volino. Journal of Physical Chemistry C, 111:3393 (2007)

[7] S. Stapf S., R. Kimmich R. and R.O. Seitter R.O., Phys. Rev. Lett., 75, 2855 (1995).

[8] S. Stapf, and R. Kimmich, Journal of Chemical Physics, 103, 2247 (1995)

[9] P. Levitz, P.G. Ehret, S.K. Sinha, J.M. Drake. J. Chem. Phys. 95, 6151 (1991)

[10] P. Levitz. Ads. Colloid. Interface Sci. 76-77, 71 (1998)

[11] P. Levitz, J.P. Korb, D. Petit, Eur. Phys. J.E.12, 29 (2003)

[12] S. Redner, A Guide to First Passage Processes, Cambridge University Press, (2001).

[13] P. Levitz, D. S. Grebenkov, M. Zinsmeister, K. Kolwankar and B. Sapoval, Phys. Rev. Lett., 96, 180601 (2006)

[14] P. Levitz, M. Zinsmeister, P. Davidson, D. Constantin and O. Poncelet,Phys. Rev. E, 78, 030102(R) (2008).

[15] B. Halle and H. Wennerstrom, J. Chem. Phys., 75, 1928 (1981)

[16] R. Kimmich, NMR tomography, Diffusimetry, Relaxometry, Springer, (1997)

[17] B. Halle, J. Chem. Phys, 94, 3150 (1991)

[18] E. M. Renkin, J. Gene. Physiology. 43, 225 (1954)

[19] M.C. Bellisent-Funel, S.H. Chen, J.M. Zanotti, Phys. Rev. E. 51, 4558 (1995).

[20] E. Steiner, S. Bouguet-Bonnet, J-L Blin, and D. Canet. J. Phys. Chem A, 115, 9941. (2011)

[21] S. Gruener, T. Hofmann, D. Wallacher, A. V. Kityk, P. Huber, Phys. Rev. E. , 79, 067301 (2009)

[22] S. Gruener, D. Wallacher, S. Greulich, M. Busch, P. Huber, Phys. Rev. E, 93,.013102 (2016) 


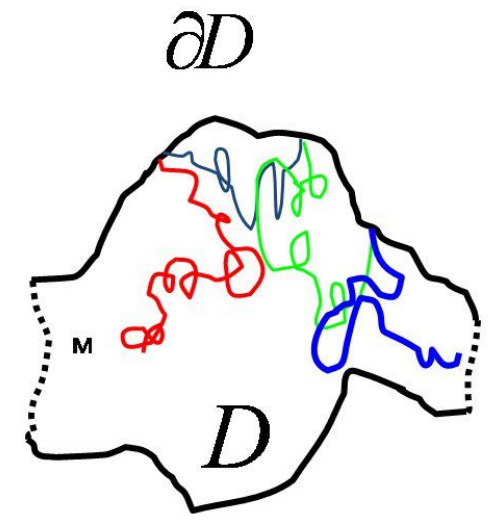

Figure 1: Intermittent dynamics near an interface $\partial D$ with a succession of adsorption steps and bridges in the pore $\mathrm{D}$. The relocation event between two successive adsorption associates several bridges 


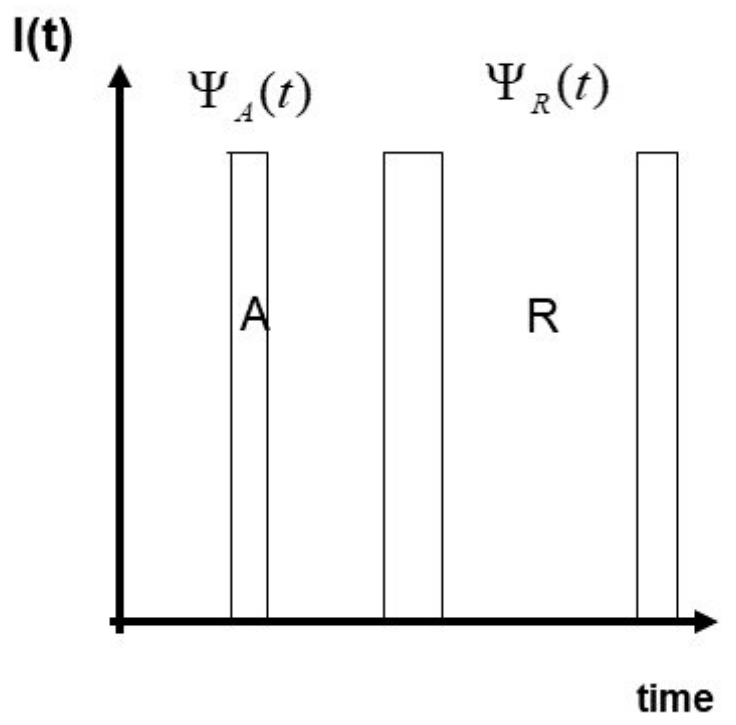

Figure 2: Evolution of the indicator function $I(t)$ equals to one in the adsorption state and zero in the confined bulk. This random signal is associated to two probability density functions, $\Psi_{\mathrm{A}}(\mathrm{t})$ and $\Psi_{\mathrm{R}}(\mathrm{t})$, describing the time distribution of adsorption and relocation events. 


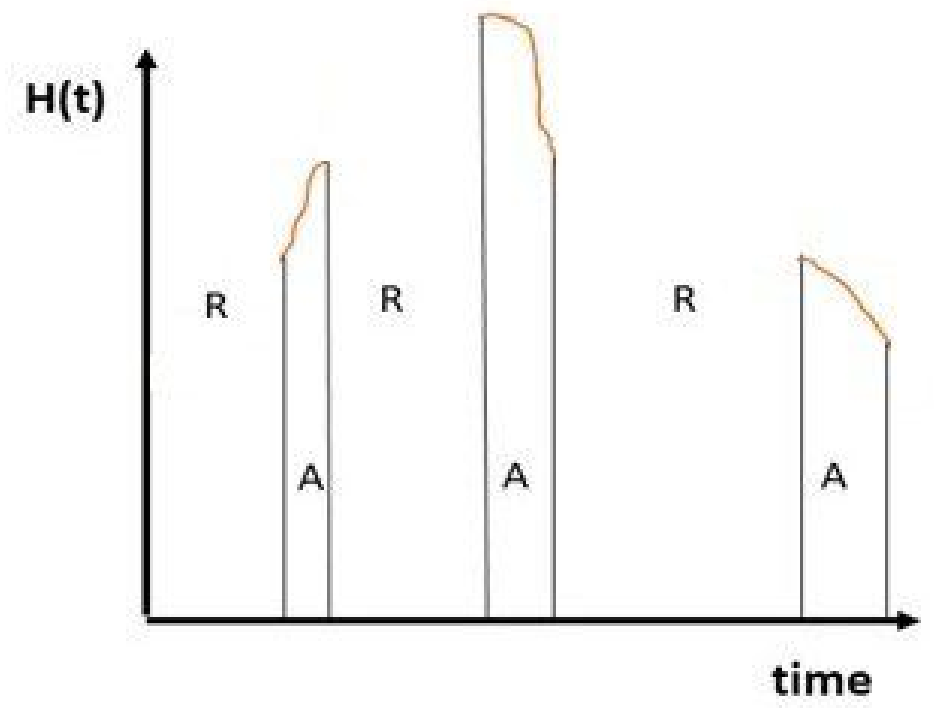

Figure 3: Evolution of the fluctuating intra-dipolar magnetic interaction $\mathrm{H}(\mathrm{t})$ during a molecular intermittent dynamic $I(t)$. This evolution is mainly driven by curvature field of poral interface coupled to molecular surface diffusion. 


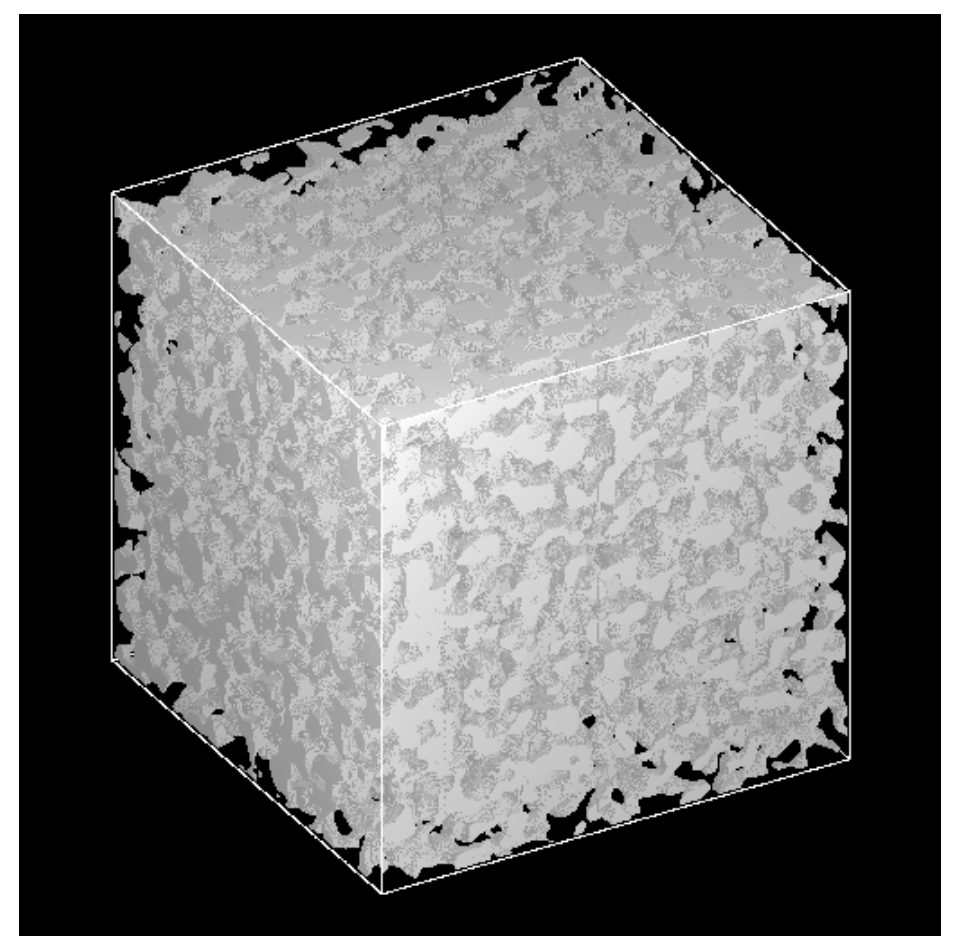

Figure 4: 3D off-lattice reconstruction of a Vycor-like porous glass. 


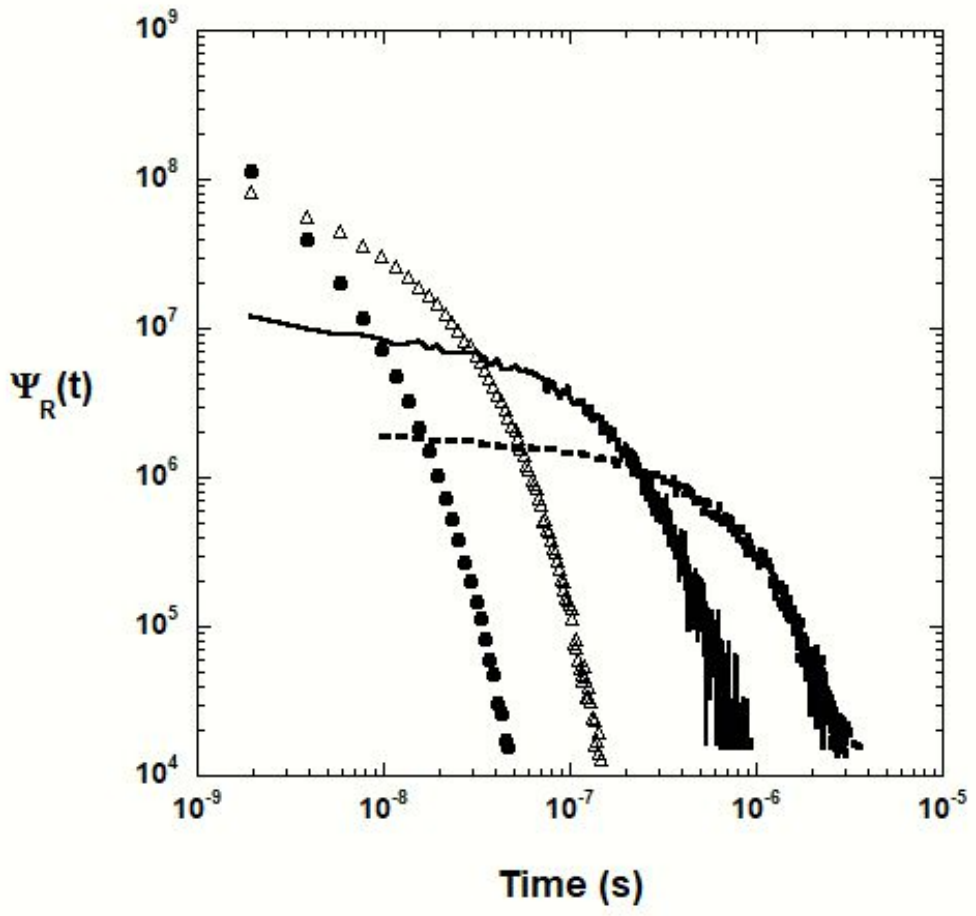

Figure 5: Evolution of the relocation p.d.f $\Psi_{\mathrm{R}}(\mathrm{t})$ with the average adsorption time $\tau_{\mathrm{A}}$. Full circles, $\tau_{A}=0.2$ ns. Open triangles, $\tau_{A}=2$ ns. Full line, $\tau_{A}=20$ ns. Dot line, $\tau_{A}=100$ ns. 


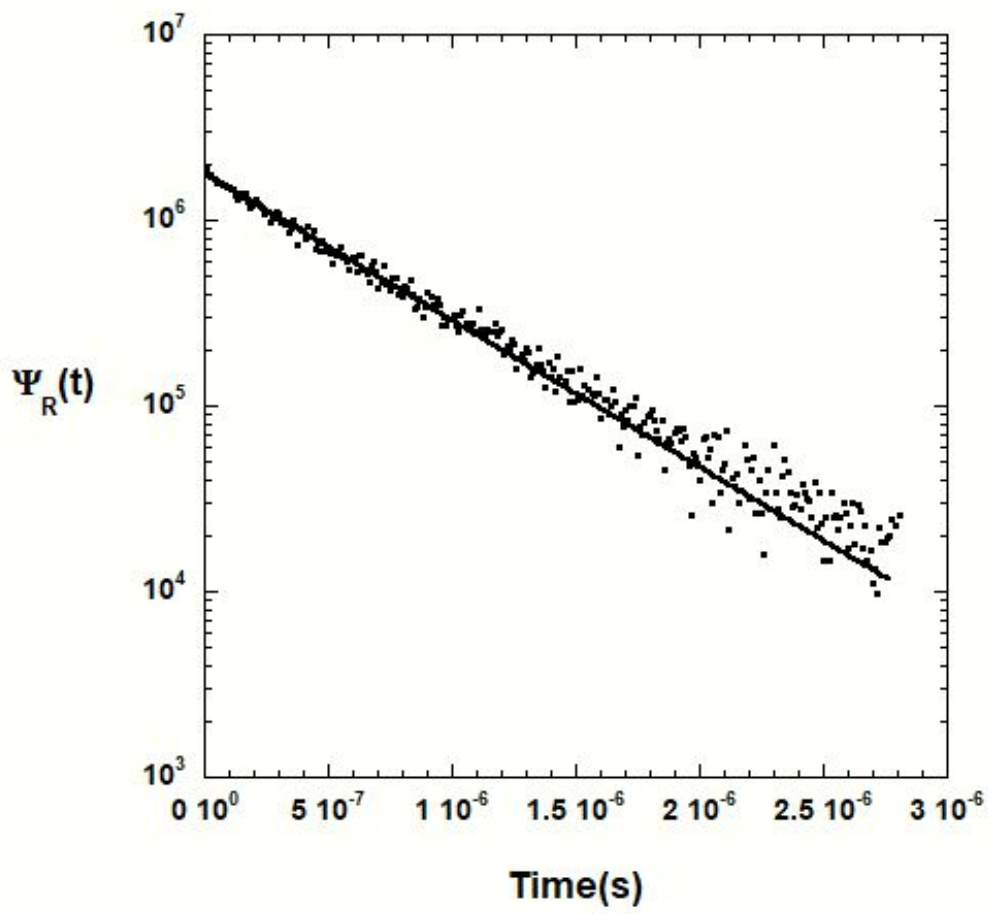

Figure 6: Evolution of the relocation p.d.f., $\Psi_{\mathrm{R}}(\mathrm{t})$, for an average adsorption time $\tau_{\mathrm{A}}=100 \mathrm{~ns}$. Full circles: numerical simulation. Continuous line: analytical prediction of $\Psi_{\mathrm{R}}(\mathrm{t})$ (using Eqs 1-3 and the reverse Fourier Transform of Eq.1). 

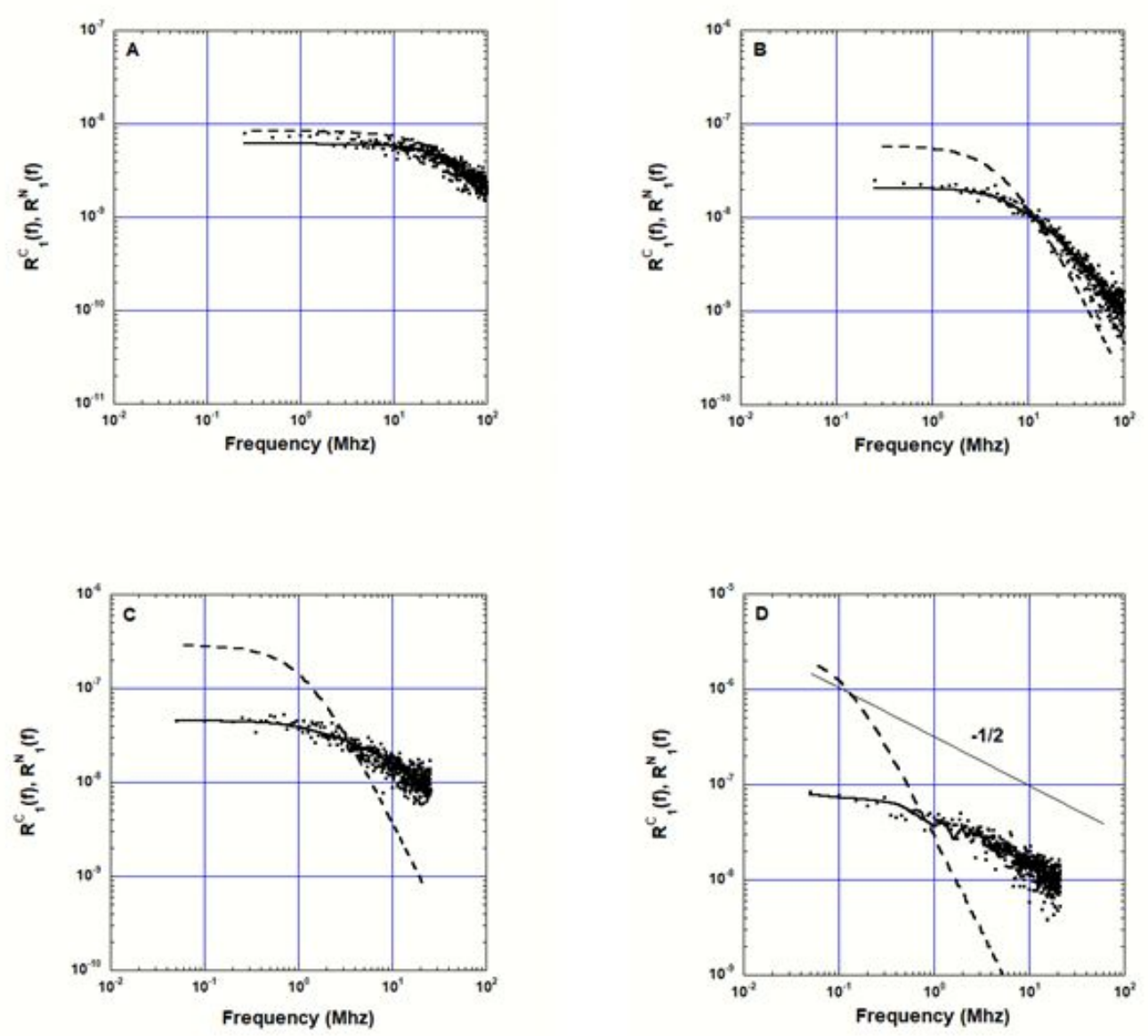

Figure 7: Frequency dependence of spin lattice relaxation rate of a molecule developing intermittent dynamics inside the Vycor-like pore network shown in Fig. 4. For the NMR relaxation process, the intramolecular dipolar interaction is considered. The average adsorption time is varied according to $2 \mathrm{~ns}$ in 4-A, $20 \mathrm{~ns}$ in 4-B, 100ns in 4-C and $2 \mu \mathrm{s}$ in 4-D. The numerical simulation using Brownian dynamics (Eqs 12-14) are represented by full squares. The dot lines are related to the computation using Eqs 5,6,7,11 (referring to an intermittence mode without surface curvature correction; Model IT). The continuous lines are related to the computation using Eqs 5,6,7,9 (referring to an interplay between surface curvature and molecular intermittence; Model IT-SC). 


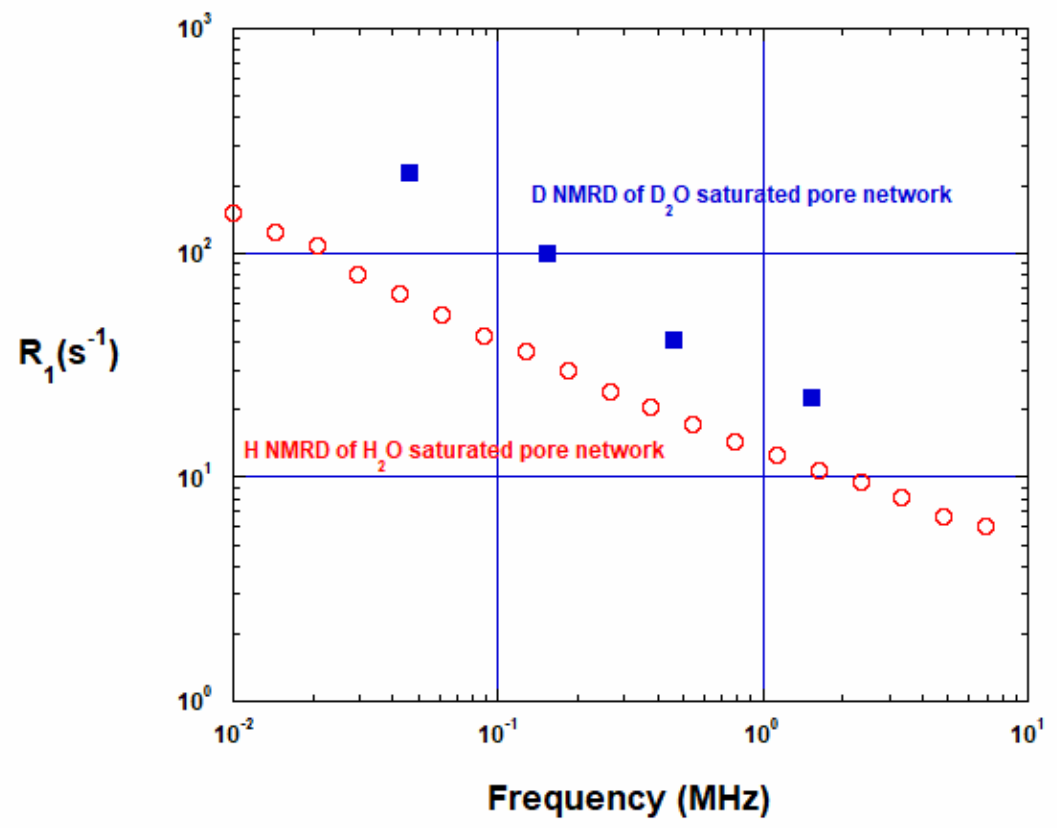

Figure 8: Water spin-lattice relaxation dispersion in fully saturated Vycor glass at $\mathrm{T}=298 \mathrm{~K}$ adapted from ref. [11]. Open circles, ${ }^{1} \mathrm{H}$ NMRD of $\mathrm{H} 20$ saturating the pore network. Full squares, ${ }^{2} \mathrm{H}$ NMRD of D20 saturating the pore network. 


\section{Graphical Abstract}

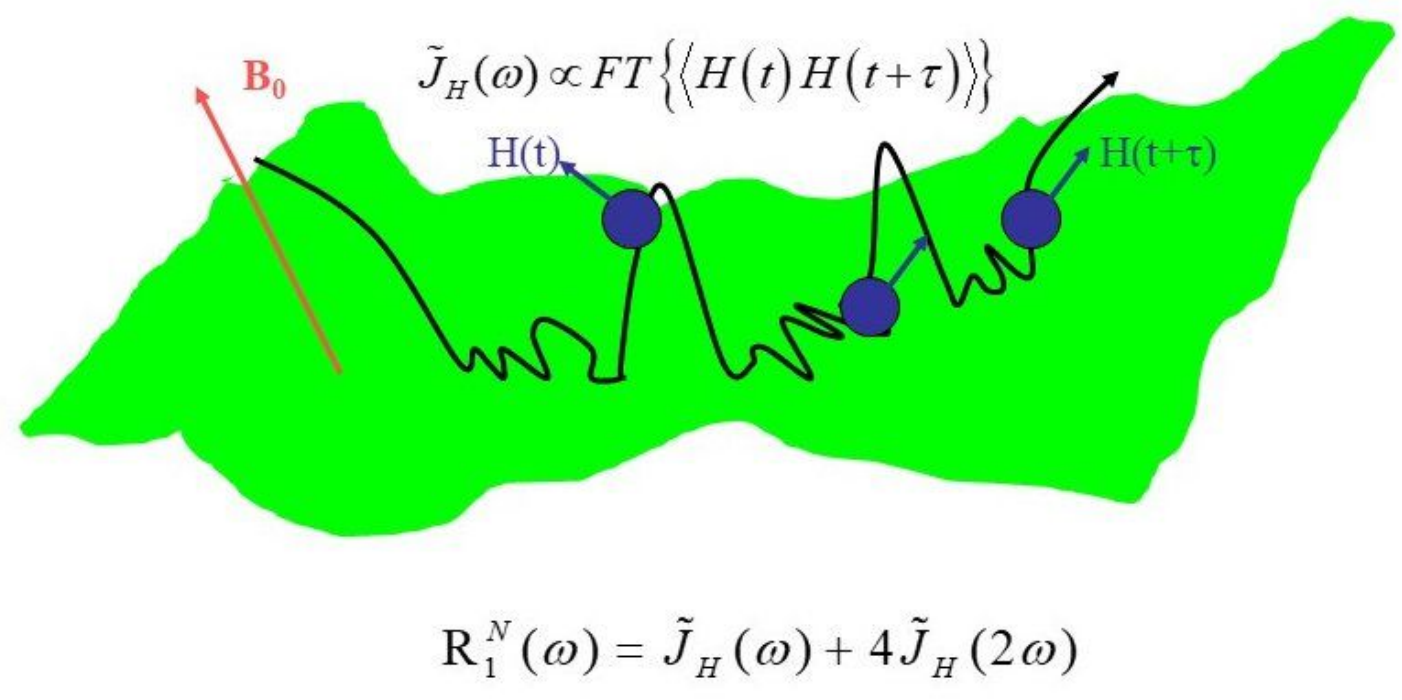

